\title{
Modification of Lethality and Mutagenesis by Growth Inhibition of Ultraviolet-irradiated Escherichia coli strain $\mathbf{B} / \mathbf{R}$
}

\author{
By RUTH F. HILL \\ Radiological Research Laboratory, Department of Radiology, \\ Columbia University, New York City, U.S.A. 10032
}

(Accepted for publication 2 January 1968)

\begin{abstract}
SUMMARY
The influence of post-irradiation growth inhibition on survival and absolute yields of prototrophic mutants has been studied in Escherichia coli $B / R$, u.v.-irradiated in the lag and logarithmic phases of the growth cycle. Without post-irradiation growth inhibition, survival and yields of mutants were higher for logarithmic phase cells. Post-irradiation growth inhibition reduced yields of mutants from both types of cells to similar extents and markedly increased the survival of logarithmic phase cells, both after small and large u.v. doses. In the case of lag phase cells, a survival increase mediated by post-irradiation growth inhibition, could only be observed by giving a large u.v. dose or by inhibiting repair of damage occurring after plating for colony formation. Post-irradiation growth inhibition was observed to have two effects-one tending to increase survival and another tending to enhance lethality. The results indicate that observable effects of postirradiation growth inhibition on survival and induced mutation in $E$. coli $B / R$ and whether or not such effects are correlated depend upon a number of factors. These include: (I) relative amounts of repair occurring during growth inhibition and during subsequent growth; (2) heterogeneity of the population with respect to growth phase, intrinsic repair ability and amount of damage; and (3) relative contributions of increased repair and increased killing - both mediated by post-irradiation growth inhibition.
\end{abstract}

\section{INTRODUCTION}

Inhibition of growth after ultraviolet (u.v.) irradiation has been used frequently to elicit information about the nature of primary damages causing lethality and about the possibility that the same primary damages also cause mutation. In Escherichia coli $\mathrm{B}$, post-irradiation growth inhibition increases survival (Roberts \& Aldous, 1949; Alper \& Gillies, 1960). The type of damage repaired is also photoreactivable, i.e. consists of u.v.-induced dimers in DNA (Castellani, Jagger \& Setlow, 1964; Cook, 1967). It is also known that in strains which are genetically defective in the ability to repair dimers by excision, u.v.-induced lethality is increased and post-irradiation growth inhibition is not very effective in improving survival (Setlow \& Carrier, 1964; Boyce \& Howard-Flanders, 1964; Harm, I966). Thus it appears that post-irradiation growth inhibition increases survival by increasing the number of dimers repaired by the excision mechanism.

Genetic reduction of the ability to excise dimers has another effect-increased sensitivity to the u.v. induction of mutations in Escherichia coli B/R (Hill, 1965; Witkin, 
I966a, $b$; Kondo \& Kato, I966). If u.v.-induced dimers are responsible for lethality in this strain as well as in $E$. coli $\mathrm{B}$, and also responsible for mutation, it might be expected that post-irradiation growth inhibition of excising strains of $B / R$ should increase survival and decrease mutation. In some cases, these expectations have been confirmed. Thus in one case where survival effects only were studied, post-irradiation growth inhibition did increase survival, although not as markedly as in $E$. coli B (Harm, I966). In two cases where both survival and mutation were determined, survival was increased and mutation decreased (Hill, 1963; Kondo \& Jagger, 1966). However, other reports have not conformed to expectation. Post-irradiation growth inhibition has failed to influence survival, although it has reduced the yield of one kind of mutation. The yield of another type of mutation in the same strain was unaffected (Witkin \& Theil, 1960). Post-irradiation growth inhibition of prototrophic $E$. coli $\mathrm{B} / \mathrm{R}$ has even reduced survival (Alper \& Gillies, 1960).

The present report concerns evidence which indicates how most of these diverse reports may be reconciled. The effects of post-irradiation growth inhibition on survival and prototrophic mutation in auxotrophic Escherichia coli $\mathbf{B} / \mathbf{R}$ were studied. The study compares results for cells irradiated in the lag and logarithmic phases of growth. Experiments were also designed to see whether post-irradiation growth inhibition might have dual effects on the same population.

\section{METHODS}

Bacterial strain. The strain employed was Escherichia coli wU 36, a tyrosine-requiring mutant of B/R. It was supplied by Dr E. M. Witkin.

Treatment of bacteria. Cultures were grown to the stationary phase by aeration in nutrient broth. To obtain cells in lag phase a $1 / 10$ dilution in broth was aerated for $45 \mathrm{~min}$; to obtain cells in logarithmic phase, a $\mathrm{I} / 2000$ broth dilution was aerated until the viable count reached $2-3 \times 10^{7}$ per $\mathrm{ml}$. (5-6 divisions). After chilling in an ice bath for $10 \mathrm{~min}$., the cultures were washed free of broth by two centrifugations and resuspended in the original volume of minimal medium $\mathrm{E}$ containing glucose (Vogel \& Bonner, 1956). Cultures were kept chilled during irradiation. For growth inhibition in liquid, irradiated cultures were rapidly diluted appropriately in this medium and held in a $37^{\circ}$ water bath. The absence of amino acids inhibits growth. One tenth $\mathrm{ml}$. samples were removed periodically and plated for viability or yields of prototrophic mutants. Experimental error was reduced by this procedure of diluting before the period of holding at $37^{\circ}$ and also by using the same $0 \cdot 1 \mathrm{ml}$. pipette for all aliquots removed from the same dilution. For growth inhibition on agar, irradiated cells were plated on minimal agar (see below). For photoreactivation, the same procedure as for growth inhibition in liquid was followed. In order to dissociate the effects of photoreactivation from those of growth inhibition in liquid, the water-bath temperature was reduced to $20^{\circ}$. As will be seen, the time for maximum photoreactivation was 5-IO min., whereas an hour was required for the maximum effect of growth inhibition in liquid.

Plating media. The standard agar medium for colony formation was SEM agar containing minimal medium E, $0.4 \%$ glucose and $5 \%$ nutrient broth. When inhibition of excision on the plate was required, acriflavine was added at a concentration of I $\mu \mathrm{g}$./ ml. (Setlow, 1964). Minimal medium agar (MM) consisted of minimal medium E, 
glucose and I $\mu$ g. tyrosine/ml. When the results of using different agar media were required, the same irradiated sample was plated on three plates of each of the different media (using the same $0.1 \mathrm{ml}$. pipette) and these platings were done in random order. Counts of auxotrophic parental colonies on SEM agar (with or without acriflavin present) were made after $24 \mathrm{hr}$ of incubation at $37^{\circ}$. Because of growth-slowing on MM agar, counts of parental colonies on this agar were made after $48 \mathrm{hr}$ of incubation. All counts of prototrophic mutant colonies were also made after $48 \mathrm{hr}$ of incubation.

Ultraviolet-irradiation and photoreactivation. The apparatus used for u.v.-irradiation, for measurement of u.v. dose and for photoreactivation (p.r.), has been described previously (Hill \& Simson, I96I ; Hill, 1965). In the present experiments, white light from the G.E. A-H 5 lamp was used for photoreactivation.

\section{RESULTS}

Figure I shows complete dose-survival and dose-mutation curves for cells irradiated in the lag and in the logarithmic phases but not subjected to post-irradiation growth inhibition, i.e. irradiation was followed by immediate plating on SEM agar. Logarithmic phase cells were-slightly more resistant to u.v.-induced lethality than lag phase cells and yielded 6-Io times more mutants. When both types of cells were irradiated with similar doses and this was followed by growth inhibition in liquid prior to plating on SEM agar, the yields of mutants were reduced at about the same rate and to about the same extent (Fig. 2).

Post-irradiation growth inhibition in liquid did not, however, have similar effects on the survival of both types of cells. In the case of lag phase cells, previous reports were confirmed in that the survival after low u.v. doses was not noticeably increased (Fig. 3) (Witkin, 1964). A survival increase-but only by a factor of two-was obtained by increasing the u.v. dose. More marked survival increases required plating on acriflavine-supplemented SEM agar (Fig. 3). In the case of logarithmic phase cells, postirradiation growth inhibition in liquid was much more effective in increasing survival. This was true for all doses and could be observed even without acriflavine supplementation of SEM agar (Fig. 4).

In the above experiments, growth inhibition was in liquid and was presumably terminated once the cells were plated on SEM agar. The marked survival increase obtained for logarithmic phase cells did not preclude the possibility that effects tending to decrease survival might also have been occurring, i.e. that the survival increase was in fact limited by such effects. Since decreased survival upon post-irradiation growth inhibition of Escherichia coli $\mathbf{B} / \mathbf{R}$ had been reported, it was decided to see whether lethal effects were also operating in the present experiments (Alper \& Gillies, 1960). For this purpose, growth inhibition in liquid of irradiated logarithmic phase cells was prolonged and the effects of subsequent plating on $\mathrm{MM}$ agar were compared with those obtained by plating on SEM agar.

Figure 5 shows the results of these experiments. Since incubation on MM agar slows growth, it was not surprising that when irradiated cells were immediately plated on this medium and allowed to form colonies on it, survival was greater than when immediate plating and colony formation were on SEM agar. The increase in survival obtained in this way was equal to the maximum increase obtained in the usual wayby growth-inhibition in liquid before plating on SEM agar. When growth-inhibition 
in liquid was prolonged before plating on SEM agar, the survival decreased again so that the gain was eventually lost. This can also be seen in Fig. 4. Thus lethal effects of growth inhibition in liquid were present. When growth inhibition was in liquid and this was followed by plating on growth-inhibiting MM agar, survival also eventually decreased. Since this manner of treating the cells amounted to even greater prolongation of growth inhibition, survival was eventually reduced below the survival obtained when

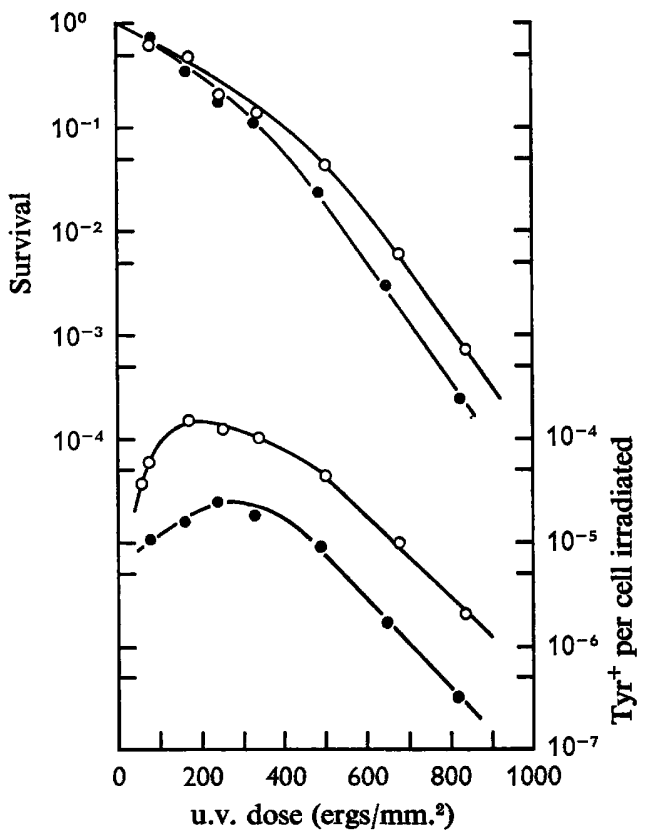

Fig. I

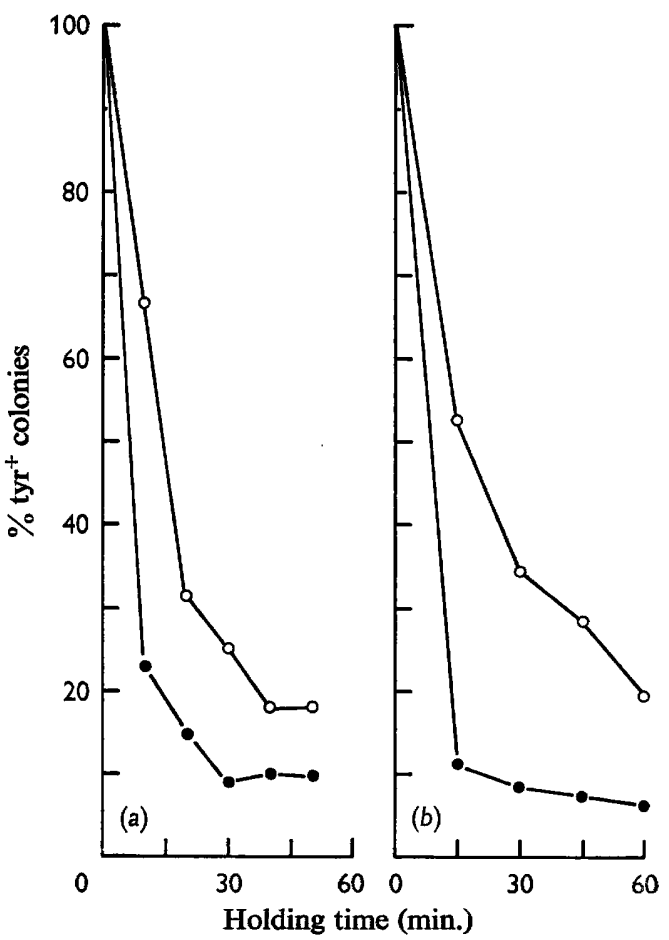

Fig. 2

Fig. I. Survival curves (above) and curves for absolute yield of tyrosine-independent mutants (below) for $E$. coli wu 36, u.v.-irradiated in lag phase (O) and in logarithmic phase (O). Irradiation followed by immediate plating on SEM agar.

Fig. 2. Effect of post-irradiation holding of cells in minimal liquid medium at $37^{\circ}$ on yields of tyr ${ }^{+}$mutants: $(a)$ cells irradiated in lag phase; $(b)$ cells irradiated in logarithmic phase. Ultraviolet doses for lag phase cells were $112 \mathrm{ergs} / \mathrm{mm}^{2}(0)$ and $430 \mathrm{ergs} / \mathrm{mm}^{2}(\mathrm{O})$; for logarithmic phase cells, I 12 ergs $/ \mathrm{mm}^{2}{ }^{2}(O)$ and $530 \mathrm{ergs} / \mathrm{mm}^{2}(O)$. Plating on SEM agar.

prolonged growth inhibition in liquid preceded plating on SEM agar. Thus lethal effects of growth inhibition occurred also on MM agar. It should be mentioned that these lethal effects were not observed in control experiments using unirradiated cells.

Lethal effects of growth inhibition in liquid have been reported to reduce photoreactivability (Castellani et al. 1964; Terry, Kilbey \& Howe, 1967). This was also observed in the present system. Irradiated logarithmic phase cells were exposed to photoreactivating light immediately after irradiation and after growth inhibition in liquid for the time required to obtain maximum survival without photoreactivation. Plating for colony formation on MM agar was compared with plating on SEM agar. 
The survival level obtained on SEM agar by growth inhibition in liquid followed by exposure to photoreactivating light was less than that obtained by photoreactivation immediately after u.v. irradiation (Fig. 6). In addition, survival after exposure to photoreactivating light was reduced if the cells were plated on MM agar rather than on SEM agar. Thus photoreactivation did not prevent the lethal effect of growth inhibition on agar.

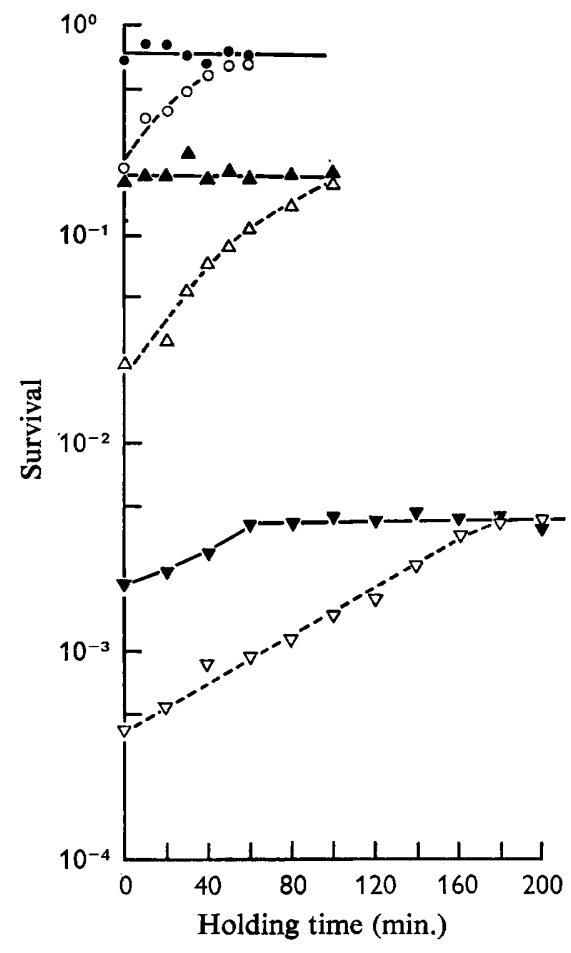

Fig. 3

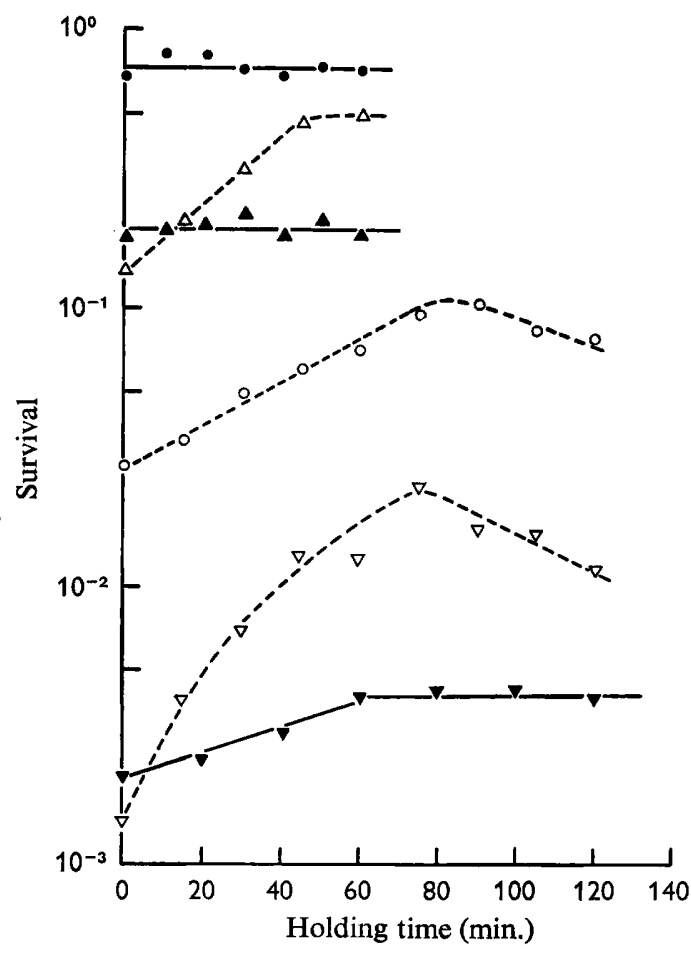

Fig. 4

Fig. 3. Effect of post-irradiation holding of cells in minimal liquid medium at $37^{\circ}$ on survival of cells irradiated in lag phase. Plating on SEM agar, - ; plating on acriflavine-SEM agar, --- . Doses were $\left.120 \mathrm{ergs} / \mathrm{mm}^{2}(\odot)\right), 280 \mathrm{ergs} / \mathrm{mm}^{2}(\Delta \Delta)$ and $660 \mathrm{ergs} / \mathrm{mm}^{2}{ }^{2}(\nabla \nabla)$.

Fig. 4. Effect of post-irradiation holding of cells in minimal liquid medium at $37^{\circ}$ on survival of cells irradiated in logarithmic phase (-- ). Data from Fig. 3 for cells irradiated in lag phase $(-)$ are repeated for comparison. Doses to logarithmic phase cells were $300 \mathrm{ergs} / \mathrm{mm} .^{2}$ $(\triangle), 520 \mathrm{ergs} / \mathrm{mm}^{2}(O)$ amd $760 \mathrm{ergs} / \mathrm{mm}^{2}(\nabla)$.

\section{DISCUSSION}

When DNA has been damaged by radiation, the survival of the cell should depend at least partly upon the repair of the damage and also upon delaying replication until the damage has been repaired. The re-initiation of DNA replication after u.v. irradiation requires prior protein synthesis but excision of u.v.-induced dimers from DNA can occur in the absence of protein synthesis (Harold \& Ziporin, 1958; Doudney, 1959; Drakulic \& Errera, 1959; Setlow, 1964). Therefore delaying the onset of protein synthesis should increase the probability of DNA repair before replication and con- 
sequently increase the probability of survival, provided that the delay does not also have adverse effects. The present results for logarithmic phase cells show that survival was indeed markedly increased, but that post-irradiation growth inhibition did have adverse effects as well. Prolongation of the inhibition revealed the existence of a lethal effect.

The rate of dimer excision in lag phase cells is poor compared with the rate for logarithmic phase cells (Setlow, 1966). Hence it is not surprising that post-irradiation growth inhibition of lag phase cells did not have the same marked effect on survival.

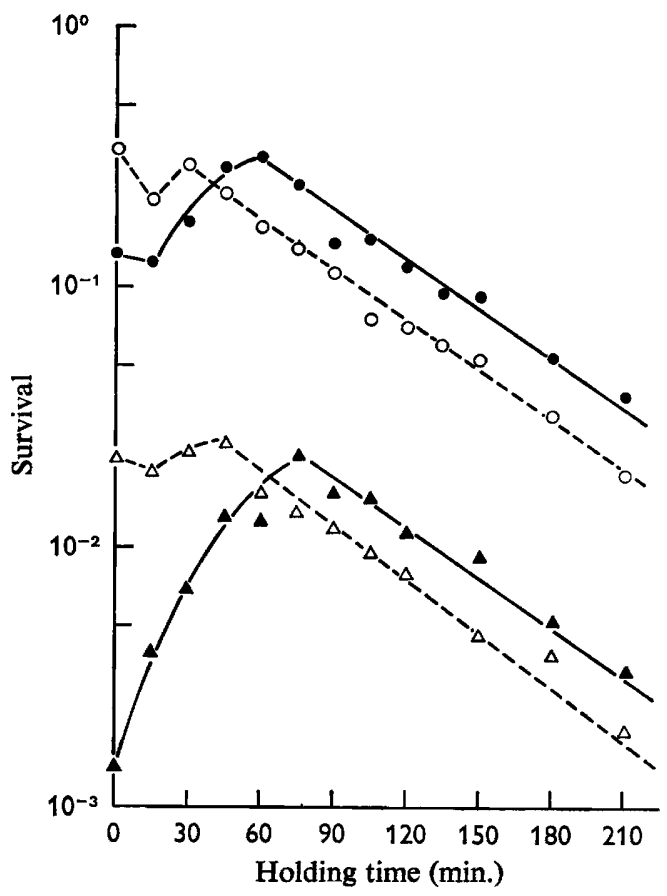

Fig. 5

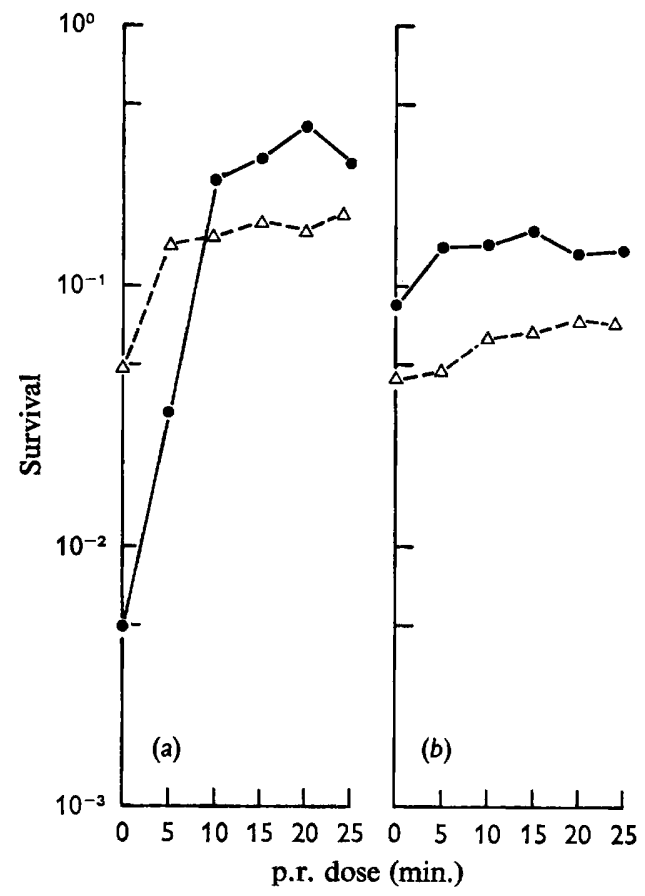

Fig. 6

Fig. 5. Effect of prolonged post-irradiation holding of cells in minimal liquid medium at $37^{\circ}$ and of subsequent plating agar on survival of logarithmic phase cells. Plating on SEM agar, -; plating on MM agar, - - . Doses were $340 \mathrm{ergs} / \mathrm{mm} .^{2}$ (upper set of curves) and $760 \mathrm{ergs} /$ mm. ${ }^{2}$ (lower set).

Fig. 6. Effect of plating agar on survival of logarithmic phase cells exposed to photoreactivating light in minimal liquid medium after u.v.-irradiation. Plating on SEM agar, -; plating on MM agar, - - - Exposure to photoreactivating light immediately after u.v.irradiation $(a)$ and after $\mathrm{I} \mathrm{hr}$ of holding in minimal liquid medium at $37^{\circ}(b)$. Temperature during exposure to photoreactivating light $=20^{\circ}$. Dose was $660 \mathrm{ergs} / \mathrm{mm} .{ }^{2}$.

However, the inhibition greatly reduced the yield of mutants from both types of cells. One reasonable explanation for this is that most of the mutants obtained from lag phase cultures may have originated in cells already in logarithmic phase, i.e. that the lag phase populations were heterogeneous in this respect. According to Fig. 2, when the same u.v. dose of $112 \mathrm{ergs} / \mathrm{mm}^{2}$ was given to both types of populations, postirradiation growth inhibition caused very nearly the same rate of loss of mutants. At this dose plating immediately after irradiation gave a tenfold greater yield of mutants from logarithmic phase cells (Fig. I). Simple calculation shows that if the presumptive 
logarithmic phase fraction in the lag phase population had amounted to only $10 \%$, it would account for nearly $100 \%$ of the mutants and thus for the rapid loss upon postirradiation growth inhibition. On the other hand, the effect of this presumptive logarithmic phase component on survival would not be nearly as great. At low doses, the increase in survival would be so small as to easily escape detection whereas at high doses, the increase would become larger and more likely to be detected (Table I).

\section{Table I. Calculation of effect of a logarithmic phase fraction in increasing survival of a lag phase population exposed to post-irradiation growth inhibition}

It is assumed that $(a)$ post-irradiation growth inhibition increases the survival of logarithmic phase cells but not lag phase cells, ${ }^{*}$ and $(b)$ the presumptive logarithmic phase fraction is $10 \%$ (see text).

$s$ = survival of logarithmic phase cells when plated immediately after irradiation;

$\mathbf{S}=$ survival of the lag phase population when plated immediately after irradiation;

$\mathrm{F}$ = factor for maximum increase in survival of logarithmic phase cells;

$F^{\prime}=$ factor for maximum increase in survival of the overall 'lag' phase population $=\frac{10^{-1} \mathrm{~S}(\mathrm{~F}-\mathrm{I})+\mathrm{S}}{\mathrm{S}}$.

Dose (ergs/mm. $\left.{ }^{2}\right)$

s (Fig. I)

S (Fig. I)

F (Fig. 4) $\dagger$

$F^{\prime}$ (calc.)

$F^{\prime}$ (obs.) (Fig. 3)
120

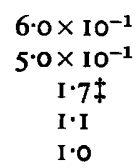

280

$2.8 \times 10^{-1}$

$2.0 \times 10^{-1}$

3.3

I 3

$1 \cdot 0$
660

$7.0 \times 10^{-3}$

$2.5 \times 10^{-8}$

$7 \cdot 5$

$2 \cdot 8$

$2 \cdot 0$

* Lag phase cells show poor dimer excision (Setlow, I966).

$\dagger$ Since the doses of Fig. 3 are not exactly the same as in Fig. 4, values for F are approximations from Fig. 4.

$\ddagger$ This assumes that at this low dose, growth inhibition rescues all logarithmic phase cells.

Another possible explanation for the marked loss of mutants, but an unimpressive survival increase upon post-irradiation growth inhibition of lag phase cells, is suggested by the lethal effect of this post-treatment. Although this was clearly demonstrated for logarithmic phase cells, it may also occur in lag phase cells. In either case, the lethal effect would limit a survival increase but would cause an even greater loss of mutants than would result from efficient dimer repair alone. It may be noted that these opposite effects of post-irradiation growth inhibition probably explain why different authors have reported different results. The net effect on survival probably varies with strain as well as with other particular experimental conditions (Kos, Drakulic \& Brdar, 1965).

Although post-irradiation growth inhibition of lag phase cells given small doses did not increase the survival measured on SEM agar, a large survival increase occurred if survival was measured on acriflavine-supplemented SEM agar. Without prior growth inhibition in liquid, survival on acriflavine-SEM was considerably lower than on SEM alone. Since acriflavine reduces excision repair, the increased lethality probably reflects loss of cells which had either more dimers to be repaired or less excision ability than cells which managed to survive in the presence of acriflavine. When plating on acriflavine-SEM was preceded by growth inhibition, the excision taking place during this inhibition was presumably sufficient to prevent loss of cells with many dimers or too little excision ability. According to this interpretation, the distribution of dimers in the population and/or the distribution of excision ability are among the 
factors which determine modification of lethality by post-irradiation treatments which modify excision repair (Hill, I965).

The fact that post-irradiation growth inhibition may have little or no detectable effect on survival but will reduce yields of prototrophic mutants has been taken as evidence that the primary u.v. lesions causing this mutation in dimer-excising strains include a lesion which is not an ordinary dimer (Witkin, 1966a). In view of the above discussion relating to other interpretations of non-correlation between effects on survival and mutant yields, it seems worthwhile to consider additional evidence which has been used to support the idea of a unique u.v. lesion for prototrophic mutation.

\section{(1) Effect of photoreactivating light}

When auxotrophic strains lacking photoreactivating enzyme were u.v.-irradiated and then exposed to photoreactivating light, the yield of prototrophic mutations was reduced, although such strains cannot split dimers (Witkin, Sicurella \& Bennett, 1963). Since the effective wavelengths of the light are known to cause growth inhibition, it would appear that this situation is covered by the previous discussion (Jagger, Wise \& Stafford, I964). Other objections to this evidence have been given (Kondo \& Jagger, 1966; Kondo \& Kato, I966).

(2) Effect of growth inhibition on $u . v .-$ induced mutation to streptomycin resistance

When u.v.-irradiated cells of a streptomycin sensitive, auxotrophic strain were kept on chloramphenicol-supplemented nutrient broth agar prior to transfer to media for scoring mutants, only the yield of prototrophic mutants was reduced (Witkin \& Theil, 1960). The scoring conditions were not, however, the same. Prototrophic mutants were scored after plating on the surface of SEM agar, streptomycin-resistant (and dependent) mutants were scored by incorporating cells within nutrient broth agar and adding streptomycin after allowing time for phenotypic expression. Therefore before this evidence can be evaluated properly it would seem necessary to know how much growth inhibition actually occurred during the exposure to chloramphenicol (stationary phase cells were used) and how much dimer repair occurred during this period relative to that occurring during the later periods of growth in different conditions. It is possible that very little dimer repair occurred during the actual period on chloramphenicol agar. The different results for the two types of mutations could then be due to a difference in the rate of chloramphenicol elimination from cells under the different scoring conditions, i.e. a slower rate on SEM agar would allow more repair on this medium and hence a much more pronounced loss of mutants.

\section{(3) Action spectra for lethality and for prototrophic mutation}

Evidence obtained from determinations of action spectra has also been quoted in support of a unique mutagenic lesion for prototrophy. The action spectrum for mutation measured on acriflavine-SEM agar has been reported to match the action spectrum for lethality but the action spectrum for mutation measured on SEM agar without acriflavine is said to differ (Witkin, 1966 b). It was suggested that on SEM agar where repair of dimers is more efficient than on acriflavine-SEM agar, most of the observable yield of mutants must come from the hypothetical second mutagenic lesion so that the mutational action spectrum as measured on SEM agar will not match the spectrum for dimer formation. This line of evidence is difficult to evaluate 
since the actual spectral data have not been published. It would be interesting to know in what way the spectrum for mutation measured on SEM agar differs from the spectrum for dimer formation and whether the discrepancy does in fact reflect a non-dimer mutagenic lesion or whether it indicates a non-mutagenic, lethal damage.

This work was performed under Contract AT-(30-I)-2740 with the U.S. Atomic Energy Commission.

\section{REFERENCES}

AlPER, T. \& GILLIES, N. E. (1960). The relationship between growth and survival after irradiation of Escherichia coli strain B and two resistant mutants. J. gen. Microbiol. 22, I 13.

Boyce, P. R. \& Howard-Flanders, P. (1964). Release of ultraviolet light-induced thymine dimers from DNA in E. coli K-12. Proc. natn. Acad. Sci., U.S.A. 5I, 293.

CAStellani, A., JAGger, J. \& SetLow, R. B. (1964). Overlap of photoreactivation and liquid holding recovery in Escherichia coli B. Science, N.Y. 143, I 170.

Cook, J. S. (1967). Direct demonstration of the monomerization of thymine-containing dimers in u.v.-irradiated DNA by yeast photoreactivating enzyme and light. Photochem. Photobiol. 6, 97.

DoudNeY, C. O. (1959). Macromolecular synthesis in bacterial recovery from ultraviolet light. Nature, Lond. 184, 189.

Drakulic, M. \& Errera, M. (1959). Chloramphenicol-sensitive DNA synthesis in normal and irradiated bacteria. Biochim. Biophys. Acta 31, 459.

HARM, W. (1966). The role of host-cell repair in liquid-holding recovery of u.v.-irradiated Escherichia coli. Photochem. Photobiol. 5, 747.

HAROLD, F. M. \& ZIPORIN, Z. Z. (1958). Synthesis of protein and DNA in Escherichia coli irradiated with ultraviolet light. Biochim. Biophys. Acta 29, 439.

HILL, R. F. (1963). Dose-mutation relationships in ultraviolet-induced reversion from auxotrophy in Escherichia coli. J. gen. Microbiol. 30, 28I.

HiLl, R. F. (1965). Ultraviolet-induced lethality and reversion to prototrophy in Escherichia coli strains with normal and reduced dark repair ability. Photochem. Photobiol. 4, 563.

HiLl, R. F. \& Simson, E. (I96I). A study of radiosensitive and radioresistant mutants of Escherichia coli strain B. J. gen. Microbiol. 24, I.

JAGGER, J., WISE, W. C. \& STAFFORD, R. S. (I964). Delay in growth and division induced by near ultraviolet radiation in Escherichia coli $\mathrm{B}$ and its role in photo-protection and liquid holding recovery. Photochem. Photobiol. 3, II.

KONDO, S. \& JAGGER, J. (1966). Action spectra for photoreactivation of mutation to prototrophy in strains of Escherichia coli possessing and lacking photoreactivating enzyme. Photochem. Photobiol. $5,189$.

KoNDO, S. \& KATO, T. (1966). Action spectra for photoreactivation of killing and mutation to prototrophy in u.v.-sensitive strains of Escherichia coli possessing and lacking photoreactivating enzyme. Photochem. Photobiol. 5, 827.

Kos, E., Drakulic, M. \& BrdaR, B. (1965). Responses of various strains of Escherichia coli to inhibition of some biosynthetic processes after ultraviolet irradiation. Nature, Lond. 205, I125.

RoBERTS, R. B. \& Aldous, E. (1949). Recovery from ultraviolet irradiation in Escherichia coli. J. Bact. $57,363$.

Setlow, R. B. (1964). Physical changes in mutagenesis. J. cell. comp. Physiol. 64 (suppl. I), 5I.

Setlow, R. B. \& CARRIER, W. L. (1964). The disappearance of thymine dimers from DNA: An error-correcting mechanism. Proc. natn. Acad. Sci., U.S.A. 51, 226.

SETLOW, J. K. (1966). The molecular basis of biological effects of ultraviolet radiation and photoreactivation. In Current Topics in Radiation Research. Ed. by M. Ebert and A. Howard. Vol. II, p. 197. Amsterdam: North-Holland Publishing Co.

TERRY, C. E., KILBEY, B. J. \& Howe, H. B. (1967). The nature of photoreactivation in Neurospora crassa. Radiation Res. 30, 739.

VogeL, H. J. \& BONNER, D. M. (1956). Acetylornithase of Escherichia coli: Partial purification and some properties. J. biol. Chem. 218, 97. 
WITKIN, E. M. (1964). Photoreversal and 'dark repair' of mutations to prototrophy induced by ultraviolet light in photoreactivable and non-photoreactivable strains of Escherichia coli. Mutation Res, I, 22.

WITKIN, E. M. (1966a). Radiation-induced mutations and their repair. Science, N.Y. 152, I345.

WrTKIN, E. M. (1966b). Mutation and the repair of radiation damage in bacteria. Radiation Res. 6, (suppl.), 30 .

WITKIN, E. M. \& THEIL, E. C. (1960). The effect of post-treatment with chloramphenicol on various ultraviolet-induced mutations in Escherichia coli. Proc. natn. Acad. Sci., U.S.A. 46, 226.

Wrtkin, E. M., Sicurella, N. A. \& BennetT, G. M. (1963). Photoreversibility of induced mutations in a non-photoreactivable strain of Escherichia coli. Proc. natn. Acad. Sci., U.S.A. 50, 1055. 